\title{
INVARIANT SETS FOR CLASSES OF MATRICES OF ZEROS AND ONES
}

\author{
RICHARD A. BRUALDI ${ }^{1}$ AND JEFFREY A. ROSS
}

\begin{abstract}
Let $\mathfrak{Y}(R, S)$ denote the class of all $m \times n$ matrices of 0 's and l's with row sum vector $R$ and column sum vector $S$. A set $I \times J(I \subseteq\{1, \ldots, m\}$, $J \subseteq\{1, \ldots, n\})$ is said to be invariant if each matrix in $\mathscr{U}(R, S)$ contains the same number of l's in the positions $I \times J$. We prove that if there are no invariant singletons, then an invariant set $I \times J$ satisfies $I=\varnothing, I=\{1, \ldots, m\}, J=\varnothing$ or $J=\{1, \ldots, n\}$.
\end{abstract}

Let $m$ and $n$ be positive integers and let $R=\left(r_{1}, \ldots, r_{m}\right)$ and $S=\left(s_{1}, \ldots, s_{n}\right)$ be nonnegative integral vectors. Let $\mathfrak{A}(R, S)$ denote the class of all $m \times n$ matrices of 0 's and 1's with row sum vector $R$ and column sum vector $S$. Conditions for $\mathfrak{A}(R, S)$ to be nonempty have been given by Gale [2] and Ryser [3], [4, p. 63]. In addition, the nonempty class $\mathfrak{U}(R, S)$ has been extensively investigated (see Chapter 6 of [4] and the references therein, see also [1]).

Let $A$ be an $m \times n$ matrix of 0 's and 1's. An interchange is a transformation which replaces the $2 \times 2$ submatrix

$$
\left[\begin{array}{ll}
1 & 0 \\
0 & 1
\end{array}\right]
$$

with the $2 \times 2$ submatrix

$$
\left[\begin{array}{ll}
0 & 1 \\
1 & 0
\end{array}\right]
$$

or vice-versa. If the submatrix (1) lies in rows $p, q$ and columns $u, v$ of $A$, then we call the interchange a $(p, q ; u, v)$-interchange. An interchange does not alter the row or column sum vectors of a matrix. Given $A, B \in \mathfrak{U}(R, S)$, Ryser [4, p. 68] has proved that there is a sequence of interchanges which transforms $A$ into $B$.

Let $A$ be an $m \times n$ matrix and let $I \subseteq\{1, \ldots, m\}, J \subseteq\{1, \ldots, n\}$. Then $A[I, J]$ denotes the submatrix of $A$ whose rows are indexed by $I$ and whose columns are indexed by $J$. If $\bar{I}=\{1, \ldots, m\}-I$ and $\bar{J}=\{1, \ldots, n\}-J$, then $A(I, J)=A[\bar{I}, \bar{J}]$. For a matrix $X$ of 0's and 1's, $\sigma(X)$ denotes the number of l's of $X$.

Consider a nonempty class $\mathfrak{A}(R, S)$ and let $I \subseteq\{1, \ldots, m\}$ and $J \subseteq$ $\{1, \ldots, n\}$. Then $I \times J$ is an invariant set for $\mathfrak{Y}(R, S)$ provided

$$
\sigma(A[I, J])=\sigma(B[I, J])
$$

Received by the editors November 1, 1979.

AMS (MOS) subject classifications (1970). Primary 05B20, 05 C50.

${ }^{1}$ Research partially supported by N.S.F. Grant No. MCS 76-06374 A01. 
for all $A, B \in \mathfrak{A}(R, S)$. Since

$$
\sigma(A(I, J))=\sum_{i \in \bar{I}} r_{i}-\sum_{j \in J} s_{j}+\sigma(A[I, J])
$$

it follows that if $I \times J$ is an invariant set for $\mathfrak{A}(R, S)$ then so is $\bar{I} \times \bar{J}$. Similarly, $I \times \bar{J}$ and $\bar{I} \times J$ are invariant sets whenever $I \times J$ is. Clearly $I \times J$ is an invariant set for $\mathfrak{A}(R, S)$ whenever one of the following holds: $I=\varnothing, I=$ $\{1, \ldots, m\}, J=\varnothing, J=\{1, \ldots, n\}$. We refer to such invariant sets as trivial; all other invariant sets are nontrivial. An invariant position is an invariant set of cardinality one. Thus the position $(i, j)$ is invariant provided all or none of the matrices in $\mathfrak{A}(R, S)$ have their $(i, j)$-entry equal to 1 . Ryser's invariant $1[4$, p. 69] corresponds to an invariant position $(i, j)$ for which the $(i, j)$-entry of each matrix in $\mathfrak{Y}(R, S)$ equals 1 .

THEOREM. Let $R=\left(r_{1}, \ldots, r_{m}\right)$ and $S=\left(s_{1}, \ldots, s_{n}\right)$ be nonnegative integral vectors such that $\mathfrak{X}(R, S)$ is nonempty. If $\mathfrak{X}(R, S)$ has a nontrivial invariant set, then it has an invariant position.

Proof. Suppose $\mathfrak{A}(R, S)$ has a nontrivial invariant set. Thus there exists an invariant set $I \times J$ such that $1<|I|<m-1,1<|J|<n-1$ and $|I|+|J|$ is maximum. If $|I|=m-1$ and $|J|=n-1$, then $(i, j)$ is an invariant position where $\bar{I}=\{i\}$ and $\bar{J}=\{j\}$. Thus we may suppose that $|J|<n-2$. Let $k \in \bar{J}$ and let $J^{\prime}=J \cup\{k\}$. Then $\left|J^{\prime}\right| \leqslant n-1$, and it follows that $I \times J^{\prime}$ is not an invariant set. Thus there exists a matrix $A=\left[a_{i j}\right] \in \mathfrak{X}(R, S)$ and a single interchange which transforms $A$ to $B$ such that

$$
\sigma\left(A\left[I, J^{\prime}\right]\right)>\sigma\left(B\left[I, J^{\prime}\right]\right)
$$

Since $I \times J$ is an invariant set, this interchange is a $(p, q ; k, l)$-interchange where $p \in I, q \in \bar{I}$, and $l \in \bar{J}^{\prime}$. By (2), $a_{p k}=1$. We partition $J$ into two sets $J_{1}, K_{1}$ such that $a_{p j}=1$ for $j \in J_{1}$ and $a_{p j}=0$ for $j \in K_{1}$. We partition $I$ into four sets $I_{1}, I_{2}, I_{3}$, $I_{4}$ and $\bar{I}$ into four sets $N_{1}, N_{2}, N_{3}, N_{4}$ such that

$$
\begin{array}{lll}
a_{i k}=1, & a_{i l}=0 & \text { for } i \in I_{1} \text { or } i \in N_{1}, \\
a_{i k}=0, & a_{i l}=1 & \text { for } i \in I_{2} \text { or } i \in N_{2}, \\
a_{i k}=1, & a_{i l}=1 & \text { for } i \in I_{3} \text { or } i \in N_{3}, \\
a_{i k}=0, & a_{i l}=0 & \text { for } i \in I_{4} \text { or } i \in N_{4} .
\end{array}
$$

Then $p \in I_{1}$ and $q \in N_{2}$.

Let $i \in N_{2}$ and suppose that $a_{i j}=0$ for some $j \in J_{1}$. Then the $(p, i ; j, l)$-interchange reduces the number of 1 's in the set $I \times J$ of positions. Since $I \times J$ is an invariant set, it follows that $A\left[N_{2}, J_{1}\right]=1$, a matrix of all 1's. In a similar way it follows that $A\left[N_{2}, K_{1}\right]=0$ and then that $A\left[I_{1}, J_{1}\right]=1$ and $A\left[I_{1}, K_{1}\right]=0$. Now let $i \in I_{2}$ and suppose that $a_{i j}=0$ for some $j \in J_{1}$. Then the $(p, i ; j, l)$-interchange followed by the $(p, q ; j, k)$-interchange increases the number of 1 's in the set of positions $I \times J$. Hence $A\left[I_{2}, J_{1}\right]=1$ and, by a similar argument, $A\left[I_{2}, K_{1}\right]=0$. Now suppose that $i \in I_{3}$ and $a_{i j}=0$ for some $j \in J_{1}$. Then the $(i, q ; j, k)$-interchange increases the number of 1 's in the set $I \times J$ of positions. It follows that 
$A\left[I_{3}, J_{1}\right]=1$. Similarly, $A\left[I_{4}, K_{1}\right]=0$. Since $\bar{I} \times J$ is also an invariant set, it follows that $A\left[N_{1}, J_{1}\right]=1, A\left[N_{1}, K_{1}\right]=0, A\left[N_{3}, J_{1}\right]=1$ and $A\left[N_{4}, K_{1}\right]=0$. Hence $A$ has the form given in (3).

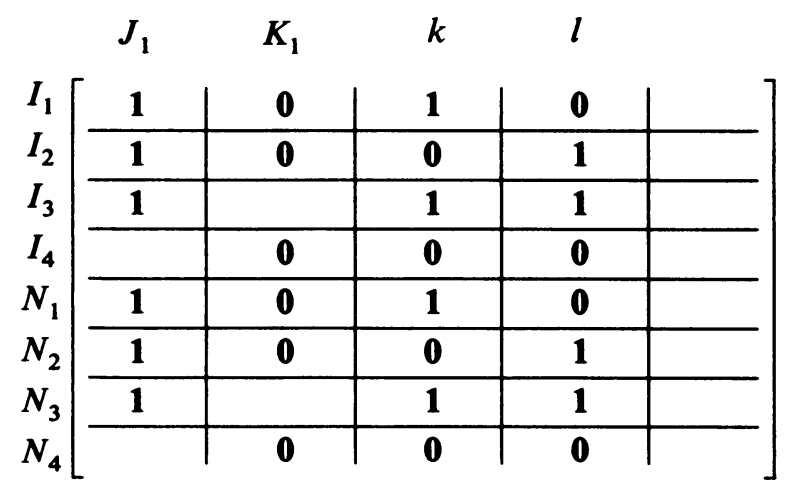

Since $J \neq \varnothing$, either $J_{1} \neq \varnothing$ or $K_{1} \neq \varnothing$. We first suppose that $K_{1} \neq \varnothing$. Define a partition $P_{1}, Q_{1}$ of $\{1, \ldots, m\}$ by

$$
P_{1}=I_{1} \cup I_{2} \cup I_{4} \cup N_{1} \cup N_{2} \cup N_{4}, \quad Q_{1}=I_{3} \cup N_{3} \text {. }
$$

Let $t>1$ and suppose sets $P_{t} \subseteq\{1, \ldots, m\}, Q_{t} \subseteq\{1, \ldots, m\}$ and $J_{t} \subseteq$ $\{1, \ldots, n\}$ have been defined. We then show how to define sets $P_{t+1}, Q_{t+1}$ and $J_{t+1}$. Let $J_{t}^{\prime}$ be the largest subset of $\{1, \ldots, n\}-\left(J_{t} \cup K_{1}\right)$ such that $A\left[Q_{t}, J_{t}^{\prime}\right]=$ 1. Let $J_{t}^{\prime \prime}$ be the subset of $\{1, \ldots, n\}$ consisting of all those $j \notin J_{t} \cup J_{t}^{\prime} \cup K_{1}$ such that $A\left[P_{t},\{j\}\right]$ contains a 1 . Then $J_{t+1}=J_{t} \cup J_{t}^{\prime} \cup J_{t}^{\prime \prime}$. Let $P_{t}^{\prime}$ be the largest subset of $Q_{t}$ such that the matrix $A\left[P_{t}^{\prime}, J_{t}^{\prime \prime}\right]$ contains a 0 in every row. For $t=0$ we define $P_{0}^{\prime}=P_{1}$. Then $P_{t+1}=P_{t} \cup P_{t}^{\prime}$ and $Q_{t+1}=Q_{t}-P_{t}^{\prime}$. It follows from the definitions that

$$
\begin{gathered}
\varnothing \neq P_{1} \subseteq P_{2} \subseteq \cdots, \\
J_{1} \subseteq J_{2} \subseteq \cdots, \\
Q_{1} \supseteq Q_{2} \supseteq \cdots, \\
P_{t}, Q_{t} \text { is a partition of }\{1, \ldots, m\}, \quad t=1,2, \ldots, \\
K_{1}, J_{t} \text { are disjoint subsets of }\{1, \ldots, n\}, \quad t=1,2, \ldots, \\
A\left[P_{t},\{1, \ldots, n\}-\left(J_{t+1} \cup K_{1}\right)\right]=0 .
\end{gathered}
$$

We have already shown that $A\left[Q_{1}, J_{1}\right]=1$. An inductive argument using the facts that $A\left[Q_{t}, J_{t}^{\prime}\right]=1$ (by choice of $J_{t}^{\prime}$ ) and $A\left[Q_{t}-P_{t}^{\prime}, J_{t}^{\prime \prime}\right]=1$ (by choice of $P_{t}^{\prime}$ ) proves that

$$
A\left[Q_{t}, J_{t}\right]=1, \quad t=1,2, \ldots
$$

We now verify the following for $t=1,2, \ldots$ by induction on $t$ :

For each matrix $B=\left[b_{r s}\right] \in \mathfrak{U}(R, S)$ such that $B\left[P_{1}, K_{1}\right]=0$ and

$$
B\left[\{1, \ldots, m\}, J_{t}\right]=A\left[\{1, \ldots, m\}, J_{t}\right],
$$

we have $B\left[P_{t}, K_{1}\right]=0$. 
For $t=1,(11)$ is trivial. Let $t>1$ and suppose (11) holds for $t$. Let $B\left[P_{1}, K_{1}\right]=$ 0 and $B\left[\{1, \ldots, m\}, J_{t+1}\right]=A\left[\{1, \ldots, m\}, J_{t+1}\right]$. Since $J_{t} \subseteq J_{t+1}$, it follows from the inductive assumption that $B\left[P_{t}, K_{1}\right]=0$. Suppose $B\left[P_{t+1}, K_{1}\right] \neq 0$. Then it follows that $B\left[P_{t}^{\prime}, K_{1}\right] \neq 0$. Thus there exist $i \in P_{t}^{\prime}$ and $j \in K_{1}$ such that $b_{i j}=1$. By definition there exists a $v \in J_{t}^{\prime \prime}$ such that $a_{i v}=0$. Since $J_{t}^{\prime \prime} \subseteq J_{t+1}$, it follows that $b_{i v}=0$. It follows from (9) and the definition of $J_{t}^{\prime \prime}$ that there exists a $u \in P_{t-1}^{\prime}$ such that $a_{u v}=1$ and hence $b_{u v}=1$. Since $B\left[P_{t}, K_{1}\right]=0$ and $P_{t-1}^{\prime} \subseteq P_{t}, b_{u j}=0$. The $(i, u ; j, v)$-interchange applied to $B$ yields a matrix $C=\left[c_{r s}\right]$ satisfying

$$
C\left[\{1, \ldots, m\}, J_{t}\right]=A\left[\{1, \ldots, m\}, J_{t}\right] \text {. }
$$

Suppose first that $t \geqslant 2$. Then $P_{1} \cap\left(P_{t}^{\prime} \cup P_{t-1}^{\prime}\right)=\varnothing$ and hence $C\left[P_{1}, K_{1}\right]=0$. It follows from the inductive assumption that $C\left[P_{t}, K_{1}\right]=0$. Since $c_{u j}=1$ where $u \in P_{t}$ and $j \in K_{1}$, we have a contradiction. Now let $t=1$. Since $k, l \in J_{1}^{\prime}$ and $j \in K_{1}$ and $v \in J_{1}^{\prime \prime}$, it follows that $C\left[\{1, \ldots, m\}, J_{1} \cup K_{1} \cup\{k, l\}\right]$ is the same as $A\left[\{1, \ldots, m\}, J_{1} \cup K_{1} \cup\{k, l\}\right]$ except that $C\left[P_{1}, K_{1}\right]$ and $A\left[P_{1}, K_{1}\right]$ differ in exactly one position as do $C\left[Q_{1}, K_{1}\right]$ and $A\left[Q_{1}, K_{1}\right]$. Thus either

$$
A\left[\{p\}, J_{1} \cup K_{1} \cup\{k, l\}\right]=C\left[\{p\}, J_{1} \cup K_{1} \cup\{k, l\}\right]
$$

or

$$
A\left[\{q\}, J_{1} \cup K_{1} \cup\{k, l\}\right]=C\left[\{q\}, J_{1} \cup K_{1} \cup\{k, l\}\right] .
$$

We are now in a position to apply our previous argument to conclude that $C$ has the form (3). Thus

$$
\mathrm{0}=C\left[P_{1}, K_{1}\right] \neq A\left[P_{1}, K_{1}\right]=\mathbf{0},
$$

a contradiction. This proves (11). In particular,

$$
A\left[P_{t}, K_{1}\right]=0, \quad t=1,2, \ldots
$$

Let $s$ be the smallest positive integer such that $J_{s}=J_{s+1}$. Then it follows that $P_{s}=P_{s+1}$ and $Q_{s}=Q_{s+1}$. Suppose $A\left[P_{s},\{1, \ldots, n\}-\left(K_{1} \cup J_{s}\right)\right] \neq 0$. Then there exists $e \in\{1, \ldots, n\}-\left(K_{1} \cup J_{s}\right)$ such that $A\left[P_{s},\{e\}\right] \neq 0$. If $A\left[Q_{s},\{e\}\right]=1$, then $e \in J_{s}^{\prime}$. If $A\left[Q_{s},\{e\}\right] \neq 1$, then $e \in J_{s}^{\prime \prime}$. Since $J_{s+1}=J_{s} \cup J_{s}^{\prime} \cup J_{s}^{\prime \prime}$, we have a contradiction and it follows that

$$
A\left[P_{s},\{1, \ldots, n\}-\left(K_{1} \cup J_{s}\right)\right]=0 .
$$

It follows from (12) and (13) that

$$
A\left[P_{s},\{1, \ldots, n\}-J_{s}\right]=0,
$$

and from (10), that $A\left[Q_{s}, J_{s}\right]=1$. Since by (7), $P_{s}, Q_{s}$ is a partition of $\{1, \ldots, m\}$, $A$ has the form

$$
\begin{gathered}
\multicolumn{2}{c}{J_{s} \bar{J}_{s}} \\
P_{s}\left[\begin{array}{l|l} 
& 0 \\
\hline Q_{s} &
\end{array}\right] .
\end{gathered}
$$

Since $p \in P_{1} \subseteq P_{s}, P_{s} \neq \varnothing$. Since we have assumed that $K_{1} \neq \varnothing$, we conclude that $\bar{J}_{s} \neq \varnothing$. It now follows from (14) that each position of the nonempty set $P_{s} \times \bar{J}_{s}$ is an invariant position of $\mathfrak{A}(R, S)$. 
A similar argument in the case that $J_{1} \neq \varnothing$ completes the proof of the theorem. We note that for $m, n>1$, the converse of the theorem follows trivially. Necessary and sufficient conditions for the class $\mathfrak{A}(R, S)$ to have an invariant position follow from Theorem 3.2 of $[4$, p. 69].

\section{REFERENCES}

1. R. A. Brualdi and J. A. Ross, On Ryser's maximum term rank formula, Linear Algebra and Appl. 29 (1980), 33-38.

2. D. Gale, A theorem on flows in networks, Pacific J. Math. 7 (1957), 1073-1082.

3. H. J. Ryser, Combinatorial properties of matrices of zeros and ones, Canad. J. Math. 9 (1957), 371-377.

4. Combinatorial mathematics, The Carus Mathematical Monographs, No. 14, The Mathematical Association of America; distributed by Wiley, New York, 1963.

Department of Mathematics, University of Wisconsin, Madison, Wisconsin 53706 (Current address of R. A. Brualdi)

Current address (J. A. Ross): Department of Mathematics, Computer Science and Statistics, University of South Carolina, Columbia, South Carolina 29208 